Encyclopedia of Algorithms 
Ming-Yang Kao (Ed.)

\section{Encyclopedia of Algorithms}

With 183 Figures and 38 Tables

With 4075 References for Further Reading

照 Springer 


\section{Ming-Yang KaO}

Professor of Computer Science

Department of Electrical Engineering and Computer Science

McCormick School of Engineering and Applied Science

Northwestern University

Evanston, IL 60208

USA

Library of Congress Control Number: 2007933824

\section{ISBN: 978-0-387-30162-4}

This publication is available also as:

Print publication under ISBN: 978-0-387-30770-1 and

Print and electronic bundle under ISBN: 978-0-387-36061-4

(C) 2008 SpringerScience+Buisiness Media, LLC.

All rights reserved. This work may not be translated or copied in whole or in part without the written permission of the publisher (Springer Science+Business Media, LLC., 233 Spring Street, New York, NY 10013, USA), except for brief excerpts in connection with reviews or scholarly analysis. Use in connection with any form of information storage and retrieval, electronic adaptation, computer software, or by similar or dissimilar methodology now known or hereafter developed is forbidden.

The use in this publication of trade names, trademarks, service marks, and similar terms, even if they are not identified as such, is not to be taken as an expression of opinion as to whether or not they are subject to proprietary rights.

springer.com

Printed on acid free paper 


\section{Preface}

The Encyclopedia of Algorithms aims to provide the researchers, students, and practitioners of algorithmic research with a mechanism to efficiently and accurately find the names, definitions, key results, and further readings of important algorithmic problems.

The work covers a wide range of algorithmic areas, and each algorithmic area is covered by a collection of entries. An encyclopedia entry is an in-depth mini-survey of an algorithmic problem and is written by an expert researcher. The entries for an algorithmic area are compiled by an area editor to survey the representative results in that area and can form the core materials of a course in the area.

The Encyclopedia does not use the format of a conventional long survey for several reasons. A conventional survey takes a handful of individuals too much time to write and is difficult to update. An encyclopedia entry contains the same kinds of information as in a conventional survey, but an encyclopedia entry is much shorter and is much easier for readers to absorb and for editors to update. Furthermore, an algorithmic area is surveyed by a collection of entries which together provide a considerable amount of up-to-date information about the area, while the writing and updating of the entries is distributed among multiple authors to speed up the work.

This reference work will be updated on a regular basis and will evolve towards primarily an Internet-based medium to allow timely updates and fast search. If you have feedback regarding a particular entry, please feel free to communicate directly with the author or the area editor of that entry. If you are interested in authoring an entry, please contact a suitable area editor. If you have suggestions on how to improve the Encyclopedia as a whole, please contact me at kao@northwestern.edu.

The credit of the Encyclopedia goes to the area editors, the entry authors, the entry reviewers, and the project editors at Springer, including Jennifer Evans and Jennifer Carlson.

Ming-Yang Kao

Editor-in-Chief 


\section{Table of Contents}

Abelian Hidden Subgroup Problem $\ldots \ldots \ldots \ldots \ldots \ldots \ldots \ldots \ldots \ldots \ldots$

1995; Kitaev

Adaptive Partitions . . . . . . . . . . . . . . . . . . . . . . . . 4 1986; Du, Pan, Shing

Adwords Pricing ................................ 7 2007; Bu, Deng, Qi

Algorithm DC-Tree for $k$ Servers on Trees $\ldots \ldots \ldots \ldots \ldots \ldots \ldots \ldots \ldots \ldots \ldots \ldots$ 1991; Chrobak, Larmore

Algorithmic Cooling . . . . . . . . . . . . . . . . . . . . . .

1999; Schulman, Vazirani

2002; Boykin, Mor, Roychowdhury, Vatan, Vrijen

Algorithmic Mechanism Design . . . . . . . . . . . . . . . . . . . . . . 16 1999; Nisan, Ronen

Algorithms for Spanners in Weighted Graphs . . . . . . . . . . . . . . . . . . . . 25

2003; Baswana, Sen

All Pairs Shortest Paths in Sparse Graphs . . . . . . . . . . . . . . . . . . . . . 28 2004; Pettie

All Pairs Shortest Paths via Matrix Multiplication . . . . . . . . . . . . . . . . . . 31 2002; Zwick

Alternative Performance Measures in Online Algorithms . . . . . . . . . . . . . . . . . 34 2000; Koutsoupias, Papadimitriou

Analyzing Cache Misses . . . . . . . . . . . . . . . . . . . . . . 37 2003; Mehlhorn, Sanders

Applications of Geometric Spanner Networks . . . . . . . . . . . . . . . . . . . . . . 40 2002; Gudmundsson, Levcopoulos, Narasimhan, Smid

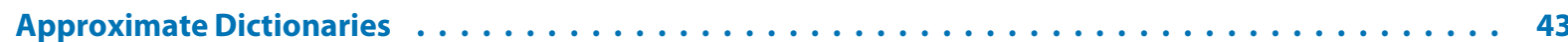

2002; Buhrman, Miltersen, Radhakrishnan, Venkatesh 
Approximate Tandem Repeats

2001; Landau, Schmidt, Sokol

2003; Kolpakov, Kucherov

Approximating Metric Spaces by Tree Metrics

1996; Bartal, Fakcharoenphol, Rao, Talwar

2004; Bartal, Fakcharoenphol, Rao, Talwar

Approximations of Bimatrix Nash Equilibria

2003; Lipton, Markakis, Mehta

2006; Daskalaskis, Mehta, Papadimitriou

2006; Kontogiannis, Panagopoulou, Spirakis

Approximation Schemes for Bin Packing .

1982; Karmarker, Karp

Approximation Schemes for Planar Graph Problems

1983; Baker

1994; Baker

Arbitrage in Frictional Foreign Exchange Market . 2003; Cai, Deng

Arithmetic Coding for Data Compression . 1994; Howard, Vitter

Assignment Problem $\ldots \ldots \ldots \ldots \ldots \ldots \ldots \ldots \ldots \ldots \ldots \ldots \ldots \ldots \ldots$

1955; Kuhn

1957; Munkres

Asynchronous Consensus Impossibility . . . . . . . . . . . . . . . . .

1985; Fischer, Lynch, Paterson

Atomic Broadcast . . . . . . . . . . . . . . . . . . . . . . .

1995; Cristian, Aghili, Strong, Dolev

Attribute-Efficient Learning

1987; Littlestone

Automated Search Tree Generation

2004; Gramm, Guo, Hüffner, Niedermeier

Backtracking Based $k$-SAT Algorithms $\ldots \ldots \ldots \ldots \ldots \ldots \ldots \ldots \ldots$

2005; Paturi, Pudlák, Saks, Zane

Best Response Algorithms for Selfish Routing . . . . . . . . . . . . . . . . . 86

2005; Fotakis, Kontogiannis, Spirakis

Bidimensionality .................................. 88

2004; Demaine, Fomin, Hajiaghayi, Thilikos

Binary Decision Graph . 
Bin Packing . . . . . . . . . . . . . . . . . . . . . . . . 94

1997; Coffman, Garay, Johnson

Boosting Textual Compression $\ldots \ldots \ldots \ldots \ldots \ldots \ldots \ldots \ldots \ldots \ldots \ldots \ldots$

2005; Ferragina, Giancarlo, Manzini, Sciortino

Branchwidth of Graphs . . . . . . . . . . . . . . . . . . . . . . . 101

2003; Fomin, Thilikos

Broadcasting in Geometric Radio Networks . . . . . . . . . . . . . . . . . . . . 105 2001; Dessmark, Pelc

B-trees ........................................ 108 1972; Bayer, McCreight

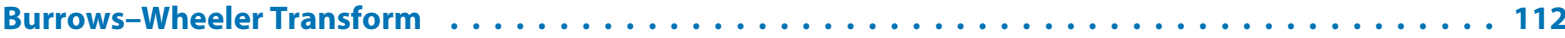
1994; Burrows, Wheeler

Byzantine Agreement . . . . . . . . . . . . . . . . . . . . . . . . . 116 1980; Pease, Shostak, Lamport

Cache-Oblivious B-Tree . . . . . . . . . . . . . . . . . . . . . . . . . . . . 121 2005; Bender, Demaine, Farach-Colton

Cache-Oblivious Model . . . . . . . . . . . . . . . . . . . . . . . . . . . 123 1999; Frigo, Leiserson, Prokop, Ramachandran

Cache-Oblivious Sorting 126 1999; Frigo, Leiserson, Prokop, Ramachandran

Causal Order, Logical Clocks, State Machine Replication 129 1978; Lamport

Certificate Complexity and Exact Learning . 131 1995; Hellerstein, Pilliapakkamnatt, Raghavan, Wilkins

Channel Assignment and Routing in Multi-Radio Wireless Mesh Networks 134 2005; Alicherry, Bhatia, Li

Circuit Partitioning: A Network-Flow-Based Balanced Min-Cut Approach 138 1994; Yang, Wong

Circuit Placement .

2000; Caldwell, Kahng, Markov

2002; Kennings, Markov

2006; Kennings, Vorwerk

Circuit Retiming .

1991; Leiserson, Saxe

Circuit Retiming: An Incremental Approach 149 2005; Zhou 
Clock Synchronization . 152

1994; Patt-Shamir, Rajsbaum

Closest String and Substring Problems 155

2002; Li, Ma, Wang

Closest Substring 156

2005; Marx

Color Coding 158 1995; Alon, Yuster, Zwick

Communication in Ad Hoc Mobile Networks Using Random Walks 161 2003; Chatzigiannakis, Nikoletseas, Spirakis

Competitive Auction . . . . . . . . . . . . . . . . . . . . . . . . . . . . 165 2001; Goldberg, Hartline, Wright

2002; Fiat, Goldberg, Hartline, Karlin

Complexity of Bimatrix Nash Equilibria 166 2006; Chen, Deng

Complexity of Core . . . . . . . . . . . . . . . . . . . . . . . . . . . . . 168 2001; Fang, Zhu, Cai, Deng

Compressed Pattern Matching $\ldots \ldots \ldots \ldots \ldots \ldots \ldots \ldots \ldots \ldots \ldots$ 2003; Kida, Matsumoto, Shibata, Takeda, Shinohara, Arikawa

Compressed Suffix Array 174 2003; Grossi, Gupta, Vitter

Compressed Text Indexing 176 2005; Ferragina, Manzini

Compressing Integer Sequences and Sets 178 2000; Moffat, Stuiver

Computing Pure Equilibria in the Game of Parallel Links 183 2002; Fotakis, Kontogiannis, Koutsoupias, Mavronicolas, Spirakis 2003; Even-Dar, Kesselman, Mansour 2003; Feldman, Gairing, Lücking, Monien, Rode

Concurrent Programming, Mutual Exclusion 188 1965; Dijkstra

Connected Dominating Set . 191 2003; Cheng, Huang, $\mathrm{Li}, \mathrm{Wu}, \mathrm{Du}$

Connectivity and Fault-Tolerance in Random Regular Graphs 195 2000; Nikoletseas, Palem, Spirakis, Yung 
Constructing a Galled Phylogenetic Network

2006; Jansson, Nguyen, Sung

CPU Time Pricing

2005; Deng, Huang, Li

Critical Range for Wireless Networks

2004; Wan, Yi

Cryptographic Hardness of Learning

1994; Kearns, Valiant

Cuckoo Hashing

2001; Pagh, Rodler

Data Migration .................................. 217

2004; Khuller, Kim, Wan

Data Reduction for Domination in Graphs . . . . . . . . . . . . . . . . . . . . . . . . . . . . . 220

2004; Alber, Fellows, Niedermeier

Decoding Reed-Solomon Codes . . . . . . . . . . . . . . . . . . . . . . . . . . . 222 1999; Guruswami, Sudan

Decremental All-Pairs Shortest Paths . . . . . . . . . . . . . . . . . . . . 226 2004; Demetrescu, Italiano

Degree-Bounded Planar Spanner with Low Weight . . . . . . . . . . . . . . . . . . . 228 2005; Song, Li, Wang

Degree-Bounded Trees 231 1994; Fürer, Raghavachari

Deterministic Broadcasting in Radio Networks 233 2000; Chrobak, Gasieniec, Rytter

Deterministic Searching on the Line 235 1988; Baeza-Yates, Culberson, Rawlins

Dictionary-Based Data Compression . 236 1977; Ziv, Lempel

Dictionary Matching and Indexing (Exact and with Errors) . . . . . . . . . . . . . . . . . . 240 2004; Cole, Gottlieb, Lewenstein

Dilation of Geometric Networks . . . . . . . . . . . . . . . . . . . . . . . . . . . . . . . . . . . 244 2005; Ebbers-Baumann, Grüne, Karpinski, Klein, Kutz, Knauer, Lingas

Directed Perfect Phylogeny (Binary Characters) $\ldots \ldots \ldots \ldots \ldots \ldots$. . . . . . . . . . . . . 246 1991; Gusfield

Direct Routing Algorithms . . . . . . . . . . . . . . . . . . . . . . . . . . 248 2006; Busch, Magdon-Ismail, Mavronicolas, Spirakis 
Distance-Based Phylogeny Reconstruction (Fast-Converging) 251

2003; King, Zhang, Zhou

Distance-Based Phylogeny Reconstruction (Optimal Radius)

1999; Atteson

2005; Elias, Lagergren

Distributed Algorithms for Minimum Spanning Trees . . . . . . . . . . . . . . . . . . . . . . 256

1983; Gallager, Humblet, Spira

Distributed Vertex Coloring

2004; Finocchi, Panconesi, Silvestri

Dynamic Trees

2005; Tarjan, Werneck

Edit Distance Under Block Operations .

2000; Cormode, Paterson, Sahinalp, Vishkin

2000; Muthukrishnan, Sahinalp

Efficient Methods for Multiple Sequence Alignment with Guaranteed Error Bounds

1993; Gusfield

Engineering Algorithms for Computational Biology .

2002; Bader, Moret, Warnow

Engineering Algorithms for Large Network Applications

2002; Schulz, Wagner, Zaroliagis

Engineering Geometric Algorithms

2004; Halperin

Equivalence Between Priority Queues and Sorting . 278 2002; Thorup

Euclidean Traveling Salesperson Problem 281 1998; Arora

Exact Algorithms for Dominating Set 284 2005; Fomin, Grandoni, Kratsch

Exact Algorithms for General CNF SAT 286 1998; Hirsch 2003; Schuler

Exact Graph Coloring Using Inclusion-Exclusion 289 2006; Björklund, Husfeldt

Experimental Methods for Algorithm Analysis 290 2001; McGeoch

External Sorting and Permuting 291 1988; Aggarwal, Vitter 
Facility Location . . . . . . . . . . . . . . . . . . . . . . . . . . . . . . . . . 299

1997; Shmoys, Tardos, Aardal

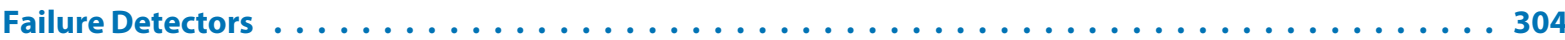
1996; Chandra, Toueg

False-Name-Proof Auction . . . . . . . . . . . . . . . . . . . . . . 308 2004; Yokoo, Sakurai, Matsubara

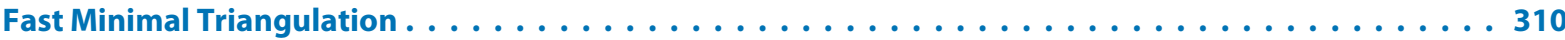
2005; Heggernes, Telle, Villanger

Fault-Tolerant Quantum Computation . . . . . . . . . . . . . . . . . . . . . 313 1996; Shor, Aharonov, Ben-Or, Kitaev

Floorplan and Placement . . . . . . . . . . . . . . . . . . . . . . 317 1994; Kajitani, Nakatake, Murata, Fujiyoshi

Flow Time Minimization . . . . . . . . . . . . . . . . . . . . . . . . . . . . . . 320 2001; Becchetti, Leonardi, Marchetti-Spaccamela, Pruhs

FPGA Technology Mapping 322 1992; Cong, Ding

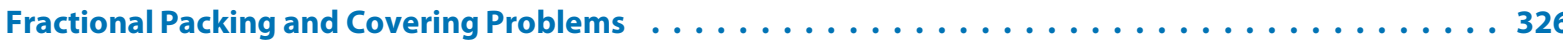
1991; Plotkin, Shmoys, Tardos 1995; Plotkin, Shmoys, Tardos

Fully Dynamic All Pairs Shortest Paths . . . . . . . . . . . . . . . . . . . . . . . . . . . . 329 2004; Demetrescu, Italiano

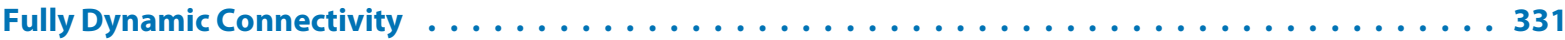
2001; Holm, de Lichtenberg, Thorup

Fully Dynamic Connectivity: Upper and Lower Bounds . . . . . . . . . . . . . . . . . . . 332 2000; Thorup

Fully Dynamic Higher Connectivity . . . . . . . . . . . . . . . . . . . . . . . 335 1997; Eppstein, Galil, Italiano, Nissenzweig

Fully Dynamic Higher Connectivity for Planar Graphs . . . . . . . . . . . . . . . . . . . . . 337 1998; Eppstein, Galil, Italiano, Spencer

Fully Dynamic Minimum Spanning Trees . . . . . . . . . . . . . . . . . . . . . . . . . 339 2000; Holm, de Lichtenberg, Thorup

Fully Dynamic Planarity Testing

1999; Galil, Italiano, Sarnak

Fully Dynamic Transitive Closure 1999; King 
General Equilibrium

2002; Deng, Papadimitriou, Safra

Generalized Steiner Network 2001; Jain

Generalized Two-Server Problem . 351 2006; Sitters, Stougie

Generalized Vickrey Auction 353 1995; Varian

Geographic Routing 355 2003; Kuhn, Wattenhofer, Zollinger

Geometric Dilation of Geometric Networks . . . . . . . . . . . . . . . . . . . . . 358 2006; Dumitrescu, Ebbers-Baumann, Grüne, Klein, Knauer, Rote

Geometric Spanners . . . . . . . . . . . . . . . . . . . . . . . . . . . . 360 2002; Gudmundsson, Levcopoulos, Narasimhan

Gomory-Hu Trees. . . . . . . . . . . . . . . . . . . . . . . . . . . . . . . 364 2007; Bhalgat, Hariharan, Kavitha, Panigrahi

Graph Bandwidth 366 1998; Feige 2000; Feige

Graph Coloring 368

1994; Karger, Motwani, Sudan 1998; Karger, Motwani, Sudan

Graph Connectivity . 371 1994; Khuller, Vishkin

Graph Isomorphism 373 1980; McKay

Greedy Approximation Algorithms 376 2004; Ruan, Du, Jia, Wu, Li, Ko

Greedy Set-Cover Algorithms . . . . . . . . . . . . . . . . . . . . . . . . . . . . 379 1974-1979, Chvátal, Johnson, Lovász, Stein

Hamilton Cycles in Random Intersection Graphs . . . . . . . . . . . . . . . . . . . . . 383 2005; Efthymiou, Spirakis

Hardness of Proper Learning . 385 1988; Pitt, Valiant

High Performance Algorithm Engineering for Large-scale Problems 387 2005; Bader 
Hospitals/Residents Problem 390

1962; Gale, Shapley

Implementation Challenge for Shortest Paths 395

2006; Demetrescu, Goldberg, Johnson

Implementation Challenge for TSP Heuristics 398

2002; Johnson, McGeoch

Implementing Shared Registers in Asynchronous Message-Passing Systems 400 1995; Attiya, Bar-Noy, Dolev

Incentive Compatible Selection 403 2006; Chen, Deng, Liu

Independent Sets in Random Intersection Graphs 405 2004; Nikoletseas, Raptopoulos, Spirakis

Indexed Approximate String Matching 408 2006; Chan, Lam, Sung, Tam, Wong

Inductive Inference

1983; Case, Smith

I/O-model .

1988; Aggarwal, Vitter

Kinetic Data Structures

1999; Basch, Guibas, Hershberger

Knapsack

1975; Ibarra, Kim

Learning with the Aid of an Oracle 1996; Bshouty, Cleve, Gavaldà, Kannan, Tamon

Learning Automata 2000; Beimel, Bergadano, Bshouty, Kushilevitz, Varricchio

Learning Constant-Depth Circuits 1993; Linial, Mansour, Nisan

Learning DNF Formulas 431 1997; Jackson

Learning Heavy Fourier Coefficients of Boolean Functions 434 1989; Goldreich, Levin

Learning with Malicious Noise 436 1993; Kearns, $L i$ 
LEDA: a Library of Efficient Algorithms

1995; Mehlhorn, Näher

Leontief Economy Equilibrium 444

2005; Codenotti, Saberi, Varadarajan, Ye 2005; Ye

Linearity Testing/Testing Hadamard Codes 446 1990; Blum, Luby, Rubinfeld

Linearizability 450 1990; Herlihy, Wing

List Decoding near Capacity: Folded RS Codes 453 2006; Guruswami, Rudra

List Scheduling 455 1966; Graham

Load Balancing 457 1994; Azar, Broder, Karlin 1997; Azar, Kalyanasundaram, Plotkin, Pruhs, Waarts

Local Alignment (with Affine Gap Weights) 459 1986; Altschul, Erickson

Local Alignment (with Concave Gap Weights) 461 1988; Miller, Myers

Local Approximation of Covering and Packing Problems 463 2003-2006; Kuhn, Moscibroda, Nieberg, Wattenhofer

Local Computation in Unstructured Radio Networks 466 2005; Moscibroda, Wattenhofer

Local Search Algorithms for $k$ SAT 468 1999; Schöning

Local Search for $\boldsymbol{K}$-medians and Facility Location 470 2001; Arya, Garg, Khandekar, Meyerson, Munagala, Pandit

Lower Bounds for Dynamic Connectivity 2004; Pătrașcu, Demaine

Low Stretch Spanning Trees . . . . . . . . . . . . . . . . . . . . . . . . . . . . 477 2005; Elkin, Emek, Spielman, Teng

LP Decoding 478 2002 and later; Feldman, Karger, Wainwright

Majority Equilibrium 
Market Games and Content Distribution 485

2005; Mirrokni

Max Cut . . 489

1994; Goemans, Williamson

1995; Goemans, Williamson

Maximum Agreement Subtree (of 2 Binary Trees) 492 1996; Cole, Hariharan

Maximum Agreement Subtree (of 3 or More Trees) 495 1995; Farach, Przytycka, Thorup

Maximum Agreement Supertree 497 2005; Jansson, Ng, Sadakane, Sung

Maximum Compatible Tree 499 2001; Ganapathy, Warnow

Maximum-Density Segment 502 1994; Huang

Maximum Matching 504 2004; Mucha, Sankowski

Maximum-scoring Segment with Length Restrictions 506 2002; Lin, Jiang, Chao

Maximum Two-Satisfiability 507 2004; Williams

Max Leaf Spanning Tree 511 2005; Estivill-Castro, Fellows, Langston, Rosamond

Metrical Task Systems . . . . . . . . . . . . . . . . . . . . . . . . . . . . . . 514 1992; Borodin, Linial, Saks

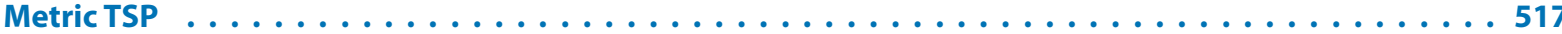
1976; Christofides

Minimum Bisection . 519 1999; Feige, Krauthgamer

Minimum Congestion Redundant Assignments 2002; Fotakis, Spirakis

Minimum Energy Broadcasting in Wireless Geometric Networks 526 2005; Ambühl

Minimum Energy Cost Broadcasting in Wireless Networks 2001; Wan, Calinescu, Li, Frieder 
Minimum Geometric Spanning Trees

1999; Krznaric, Levcopoulos, Nilsson

Minimum $\boldsymbol{k}$-Connected Geometric Networks

2000; Czumaj, Lingas

Minimum Makespan on Unrelated Machines

1990; Lenstra, Shmoys, Tardos

Minimum Spanning Trees

2002; Pettie, Ramachandran

Minimum Weighted Completion Time . 1999; Afrati et al.

Minimum Weight Triangulation . 546 1998; Levcopoulos, Krznaric

Mobile Agents and Exploration 548 1952; Shannon

Multicommodity Flow, Well-linked Terminals and Routing Problems 551 2005; Chekuri, Khanna, Shepherd

Multicut 554 1993; Garg, Vazirani, Yannakakis 1996; Garg, Vazirani, Yannakakis

Multidimensional Compressed Pattern Matching . . . . . . . . . . . . . . . . 556 2003; Amir, Landau, Sokol

Multidimensional String Matching . . 559 1999; Kärkkäinen, Ukkonen

Multi-level Feedback Queues 562 1968; Coffman, Kleinrock

Multiple Unit Auctions with Budget Constraint 563 2005; Borgs, Chayes, Immorlica, Mahdian, Saberi 2006; Abrams

Multiplex PCR for Gap Closing (Whole-genome Assembly) 565 2002; Alon, Beigel, Kasif, Rudich, Sudakov

Multiway Cut 567 1998; Calinescu, Karloff, Rabani

Nash Equilibria and Dominant Strategies in Routing 571 2005; Wang, $L i$, Chu 
Negative Cycles in Weighted Digraphs 576

1994; Kavvadias, Pantziou, Spirakis, Zaroliagis

Non-approximability of Bimatrix Nash Equilibria .

578

2006; Chen, Deng, Teng

Non-shared Edges

579 1985; Day

Nucleolus 581 2006; Deng, Fang, Sun

Oblivious Routing . 585 2002; Räcke

Obstacle Avoidance Algorithms in Wireless Sensor Networks 588 2007; Powell, Nikoletseas

$O(\log \log n)$-competitive Binary Search Tree 592 2004; Demaine, Harmon, Iacono, Patrascu

Online Interval Coloring 594 1981; Kierstead, Trotter

Online List Update 598 1985; Sleator, Tarjan

Online Paging and Caching . 601 1985-2002; multiple authors

Optimal Probabilistic Synchronous Byzantine Agreement 604 1988; Feldman, Micali

Optimal Stable Marriage 606 1987; Irving, Leather, Gusfield

P2P 611 2001; Stoica, Morris, Karger, Kaashoek, Balakrishnan

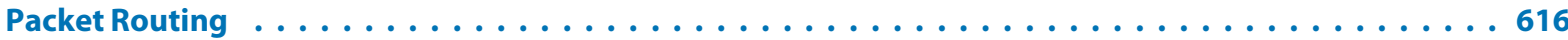
1988; Leighton, Maggs, Rao

Packet Switching in Multi-Queue Switches 618 2004; Azar, Richter; Albers, Schmidt

Packet Switching in Single Buffer 621 2003; Bansal, Fleischer, Kimbrel, Mahdian, Schieber, Sviridenko

PAC Learning . 622 1984; Valiant 
Paging 625

1985; Sleator, Tarjan, Fiat, Karp, Luby, McGeoch, Sleator, Young

1991; Sleator, Tarjan; Fiat, Karp, Luby, McGeoch, Sleator, Young

Parallel Algorithms for Two Processors Precedence Constraint Scheduling 627

2003; Jung, Serna, Spirakis

Parallel Connectivity and Minimum Spanning Trees . 629 2001; Chong, Han, Lam

Parameterized Algorithms for Drawing Graphs 631 2004; Dujmovic, Whitesides

Parameterized Matching 635 1993; Baker

Parameterized SAT . 639 2003; Szeider

Peptide De Novo Sequencing with MS/MS 640 2005; Ma, Zhang, Liang

Perceptron Algorithm . 642 1959; Rosenblatt

Perfect Phylogeny (Bounded Number of States) 644 1997; Kannan, Warnow

Perfect Phylogeny Haplotyping 647 2005; Ding, Filkov, Gusfield

Performance-Driven Clustering . 650 1993; Rajaraman, Wong

Phylogenetic Tree Construction from a Distance Matrix 651 1989; Hein

Planar Geometric Spanners 653 2005; Bose, Smid, Gudmundsson

Planarity Testing 656 1976; Booth, Lueker

Point Pattern Matching 657 2003; Ukkonen, Lemström, Mäkinen

Position Auction . 660 2005; Varian

Predecessor Search 661 2006; Pătraşcu, Thorup

Price of Anarchy 665 2005; Koutsoupias 
Price of Anarchy for Machines Models . 667 2002; Czumaj, Vöcking

Probabilistic Data Forwarding in Wireless Sensor Networks . 671 2004; Chatzigiannakis, Dimitriou, Nikoletseas, Spirakis

Quantization of Markov Chains 677 2004; Szegedy

Quantum Algorithm for Checking Matrix Identities 680 2006; Buhrman, Spalek

Quantum Algorithm for the Collision Problem 682 1998; Brassard, Hoyer, Tapp

Quantum Algorithm for the Discrete Logarithm Problem 1994; Shor

Quantum Algorithm for Element Distinctness . . . . . . . . . . . . . . . . . . . . . . . . 686 2004; Ambainis

Quantum Algorithm for Factoring 689 1994; Shor

Quantum Algorithm for Finding Triangles 690 2005; Magniez, Santha, Szegedy

Quantum Algorithm for the Parity Problem 693 1985; Deutsch

Quantum Algorithms for Class Group of a Number Field 694 2005; Hallgren

Quantum Algorithm for Search on Grids 696 2005; Ambainis, Kempe, Rivosh

Quantum Algorithm for Solving the Pell's Equation 698 2002; Hallgren

Quantum Approximation of the Jones Polynomial . . . . . . . . . . . . . . . . . . . . . 700 2005; Aharonov, Jones, Landau

Quantum Dense Coding

1992; Bennett, Wiesner

Quantum Error Correction 705 1995; Shor

Quantum Key Distribution 708 1984; Bennett, Brassard 1991; Ekert 
Quorums

1985; Garcia-Molina, Barbara

Radiocoloring in Planar Graphs

2005; Fotakis, Nikoletseas, Papadopoulou, Spirakis

Randomization in Distributed Computing 723

1996; Chandra

Randomized Broadcasting in Radio Networks

1992; Reuven Bar-Yehuda, Oded Goldreich, Alon Itai

Randomized Energy Balance Algorithms in Sensor Networks 728

2005; Leone, Nikoletseas, Rolim

Randomized Gossiping in Radio Networks .

2001; Chrobak, Gasieniec, Rytter

Randomized Minimum Spanning Tree

1995; Karger, Klein, Tarjan

Randomized Parallel Approximations to Max Flow

1991; Serna, Spirakis

Randomized Rounding

1987; Raghavan, Thompson

Randomized Searching on Rays or the Line

1993; Kao, Reif, Tate

Random Planted 3-SAT

2003; Flaxman

Ranked Matching

2005; Abraham, Irving, Kavitha, Mehlhorn

Rank and Select Operations on Binary Strings

1974; Elias

Rate-Monotonic Scheduling 751 1973; Liu, Layland

Rectilinear Spanning Tree 2002; Zhou, Shenoy, Nicholls

Rectilinear Steiner Tree 2004; Zhou

Registers

1986; Lamport, Vitanyi, Awerbuch 
2004; Navarro, Raffinot

Reinforcement Learning

1992; Watkins

Renaming .

1990; Attiya, Bar-Noy, Dolev, Peleg, Reischuk

RNA Secondary Structure Boltzmann Distribution

777

2005; Miklós, Meyer, Nagy

RNA Secondary Structure Prediction Including Pseudoknots

780

2004; Lyngsø

RNA Secondary Structure Prediction by Minimum Free Energy

782

2006; Ogurtsov, Shabalina, Kondrashov, Roytberg

Robotics .

1997; (Navigation) Blum, Raghavan, Schieber

1998; (Exploration) Deng, Kameda, Papadimitriou

2001; (Localization) Fleischer, Romanik, Schuierer, Trippen

Robust Geometric Computation

788

2004; Li, Yap

Routing

791

2003; Azar, Cohen, Fiat, Kaplan, Räcke

Routing in Geometric Networks . . . . . . . . . . . . . . . . . . . . . . . . . . . . . . . . . . . 793

2003; Kuhn, Wattenhofer, Zhang, Zollinger

Routing in Road Networks with Transit Nodes . . . . . . . . . . . . . . . . . . . . . 796

2007; Bast, Funke, Sanders, Schultes

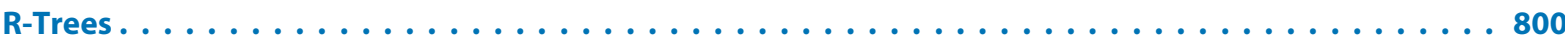

2004; Arge, de Berg, Haverkort, Yi

Schedulers for Optimistic Rate Based Flow Control . . . . . . . . . . . . . . . . . . 803 2005; Fatourou, Mavronicolas, Spirakis

Scheduling with Equipartition . . . . . . . . . . . . . . . . . . . . . . . . . 806 2000; Edmonds

Selfish Unsplittable Flows: Algorithms for Pure Equilibria . . . . . . . . . . . . . . . . . . . 810 2005; Fotakis, Kontogiannis, Spirakis

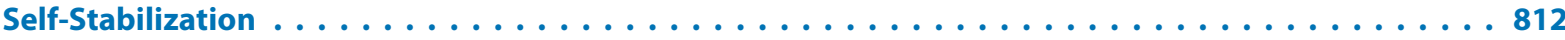
1974; Dijkstra

Separators in Graphs 
Sequential Approximate String Matching

2003; Crochemore, Landau, Ziv-Ukelson

2004; Fredriksson, Navarro

Sequential Circuit Technology Mapping 820

1998; Pan, Liu

Sequential Exact String Matching 824

1994; Crochemore, Czumaj, Gasieniec, Jarominek, Lecroq, Plandowski, Rytter

Sequential Multiple String Matching

1999; Crochemore, Czumaj, Gasieniec, Lecroq, Plandowski, Rytter

Set Agreement .

1993; Chaudhuri

Set Cover with Almost Consecutive Ones

2004; Mecke, Wagner

Shortest Elapsed Time First Scheduling

2003; Bansal, Pruhs

Shortest Paths Approaches for Timetable Information

2004; Pyrga, Schulz, Wagner, Zaroliagis

Shortest Paths in Planar Graphs with Negative Weight Edges

2001; Fakcharoenphol, Rao

Shortest Vector Problem

1982; Lenstra, Lenstra, Lovasz

Similarity between Compressed Strings

2005; Kim, Amir, Landau, Park

Single-Source Fully Dynamic Reachability 846 2005; Demetrescu, Italiano

Single-Source Shortest Paths 1999; Thorup

Ski Rental Problem

1990; Karlin, Manasse, McGeogh, Owicki

Slicing Floorplan Orientation

1983; Stockmeyer

Snapshots in Shared Memory

1993; Afek, Attiya, Dolev, Gafni, Merritt, Shavit

Sorting Signed Permutations by Reversal (Reversal Distance) 858

2001; Bader, Moret, Yan

Sorting Signed Permutations by Reversal (Reversal Sequence) 
Sorting by Transpositions and Reversals (Approximate Ratio 1.5) 863 2004; Hartman, Sharan

Sparse Graph Spanners 867 2004; Elkin, Peleg

Sparsest Cut 868 2004; Arora, Rao, Vazirani

Speed Scaling 870 1995; Yao, Demers, Shenker

Sphere Packing Problem . 871 2001; Chen, Hu, Huang, Li, Xu

Squares and Repetitions 874 1999; Kolpakov, Kucherov

Stable Marriage 877 1962; Gale, Shapley

Stable Marriage and Discrete Convex Analysis 880 2000; Eguchi, Fujishige, Tamura, Fleiner

Stable Marriage with Ties and Incomplete Lists 883 2007; Iwama, Miyazaki, Yamauchi

Stable Partition Problem 885 2002; Cechlárová, Hajduková

Stackelberg Games: The Price of Optimum 888 2006; Kaporis, Spirakis

Statistical Multiple Alignment 892 2003; Hein, Jensen, Pedersen

Statistical Query Learning 894 1998; Kearns

Steiner Forest 897 1995; Agrawal, Klein, Ravi

Steiner Trees 900 2006; Du, Graham, Pardalos, Wan, Wu, Zhao

Stochastic Scheduling 904 2001; Glazebrook, Nino-Mora

String Sorting 907 1997; Bentley, Sedgewick 
Succinct Data Structures for Parentheses Matching . 912 2001; Munro, Raman

Succinct Encoding of Permutations: Applications to Text Indexing 915 2003; Munro, Raman, Raman, Rao

Suffix Array Construction 919 2006; Kärkkäinen, Sanders, Burkhardt

Suffix Tree Construction in Hierarchical Memory 922 2000; Farach-Colton, Ferragina, Muthukrishnan

Suffix Tree Construction in RAM 925 1997; Farach-Colton

Support Vector Machines 928 1992; Boser, Guyon, Vapnik

Symbolic Model Checking 932 1990; Burch, Clarke, McMillan, Dill

Synchronizers, Spanners 935 1985; Awerbuch

Table Compression 939 2003; Buchsbaum, Fowler, Giancarlo

Tail Bounds for Occupancy Problems 942 1995; Kamath, Motwani, Palem, Spirakis

Technology Mapping 944 1987; Keutzer

Teleportation of Quantum States 947 1993; Bennett, Brassard, Crepeau, Jozsa, Peres, Wootters

Text Indexing 950 1993; Manber, Myers

Thresholds of Random $\boldsymbol{k}$-SAT . 954 2002; Kaporis, Kirousis, Lalas

Topology Approach in Distributed Computing 956 1999; Herlihy Shavit

Trade-Offs for Dynamic Graph Problems 958 2005; Demetrescu, Italiano

Traveling Sales Person with Few Inner Points 961 2004; Deìneko, Hoffmann, Okamoto, Woeginger 
Treewidth of Graphs 968

1987; Arnborg, Corneil, Proskurowski

Truthful Mechanisms for One-Parameter Agents 970 2001; Archer, Tardos

Truthful Multicast .

2004; Wang, Li, Wang

TSP-Based Curve Reconstruction 976 2001; Althaus, Mehlhorn

Two-Dimensional Pattern Indexing 979 2005; Na, Giancarlo, Park

Two-Dimensional Scaled Pattern Matching 982 2006; Amir, Chencinski

Two-Interval Pattern Problems 985 2004; Vialette 2007; Cheng, Yang, Yuan

Two-Level Boolean Minimization 989 1956; McCluskey

Undirected Feedback Vertex Set 995 2005; Dehne, Fellows, Langston, Rosamond, Stevens; 2005; Guo, Gramm, Hüffner, Niedermeier, Wernicke

Utilitarian Mechanism Design for Single-Minded Agents . 2005; Briest, Krysta, Vöcking

Vertex Cover Kernelization . .1003

2004; Abu-Khzam, Collins, Fellows, Langston, Suters, Symons

Vertex Cover Search Trees .1006

2001; Chen, Kanj, Jia

Visualization Techniques for Algorithm Engineering .1008 2002; Demetrescu, Finocchi, Italiano, Näher

Voltage Scheduling . .1011 2005; Li, Yao

Wait-Free Synchronization .1015 1991; Herlihy

Weighted Connected Dominating Set .1020 2005; Wang, Wang, Li 
Weighted Random Sampling .1024 2005; Efraimidis, Spirakis

Well Separated Pair Decomposition .1027 2003; Gao, Zhang

Well Separated Pair Decomposition for Unit-Disk Graph . .1030 1995; Callahan, Kosaraju

Wire Sizing 1999; Chu, Wong

Work-Function Algorithm for k Servers . .1035 1994; Koutsoupias, Papadimitriou

Chronological Index . . . . . . . . . . . . . . . . . . . . . . 1039

Bibliography . . . . . . . . . . . . . . . . . . . . . . 1053

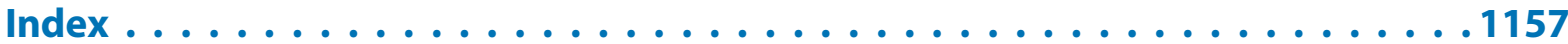




\section{About the Editor}

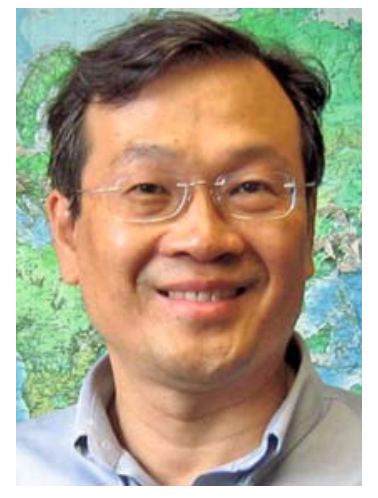

Ming-Yang Kao is a Professor of Computer Science in the Department of Electrical Engineering and Computer Science at Northwestern University. He has published extensively in the design, analysis, and applications of algorithms. His current interests include discrete optimization, bioinformatics, computational economics, computational finance, and nanotechnology. He serves as the Editor-in-Chief of Algorithmica.

He obtained a B.S. in Mathematics from National Taiwan University in 1978 and a Ph.D. in Computer Science from Yale University in 1986. He previously taught at Indiana University at Bloomington, Duke University, Yale University, and Tufts University. At Northwestern University, he has served as the Department Chair of Computer Science. He has also co-founded the Program in Computational Biology and Bioinformatics and served as its Director. He currently serves as the Head of the EECS Division of Computing, Algorithms, and Applications and is a member of the Theoretical Computer Science Group.

For more information please see: www.cs.northwestern.edu/ kao 


\section{Area Editors}

Online Algorithms Approximation Algorithms

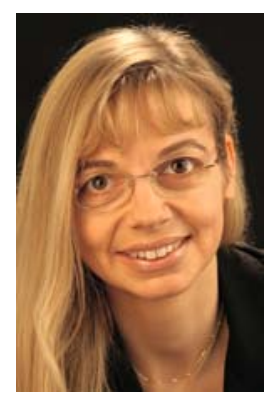

Albers, Susanne

University of Freiburg

Freiburg

Germany
External Memory Algorithms and Data Structures

Cache-Oblivious Algorithms and Data Structures

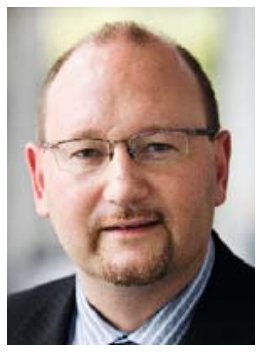

ARGe, LARS

University of Aarhus

Aarhus

Denmark

Mechanism Design

Online Algorithms

Price of Anarchy

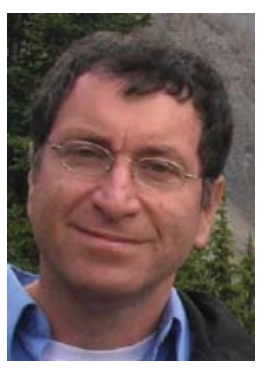

AZAR, YOSSI

Tel-Aviv University

Tel-Aviv

Israel
AmbAINIS, ANDRIS

University of Latvia

Riga

Latvia (c) University of Latvia

Press Center 


\section{Approximation Algorithms}

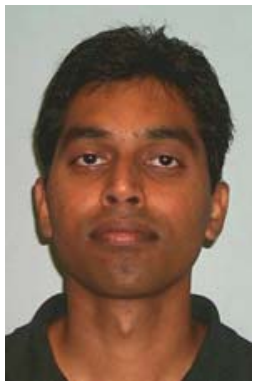

CHEKURI, CHANDRA

University of Illinois, Urbana-Champaign Urbana, IL

USA

\section{Online Algorithms}

Radio Networks

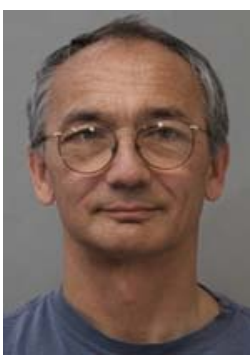

CHROBAK, MAREK

University of California, Riverside

Riverside, CA

USA

Internet Algorithms

Network and Communication Protocols

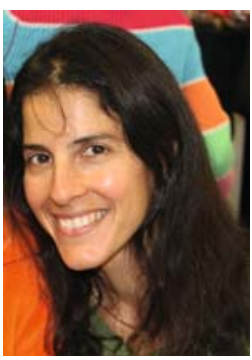

Cohen, Edith

AT\&T Labs

Florham Park, NJ

USA

\section{Bioinformatics}

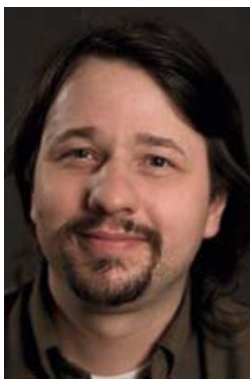

CSÜRÖS, MIKLÓS

University of Montreal

Montreal, QC

Canada

\section{Computational Economics}

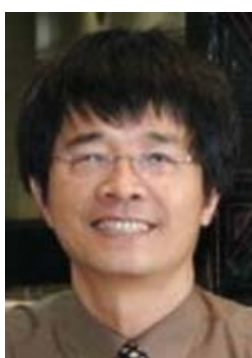

Deng, Xiaotie

University of Hong Kong

Hong Kong

China

Combinatorial Group Testing Mathematical Optimization Steiner Tree Algorithms

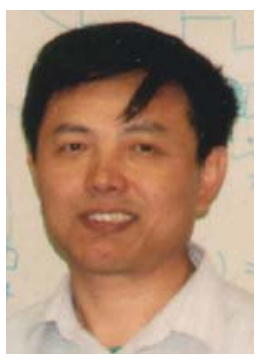

DU, DING-ZHU

University of Texas, Dallas

Richardson, TX

USA 
String Algorithms and Data Structures Data Compression

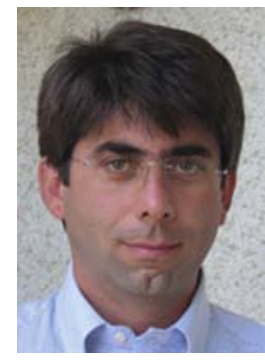

Ferragina, PaOlo

University of Pisa

Pisa

Italy

\section{Coding Algorithms}

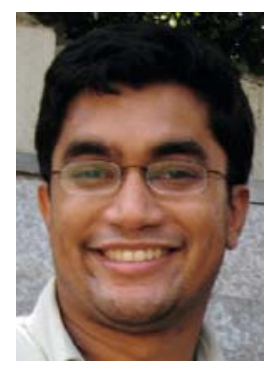

Guruswami, Venkatesan

University of Washington

Seattle, WA

USA

\section{Algorithm Engineering} Dynamic Graph Algorithms

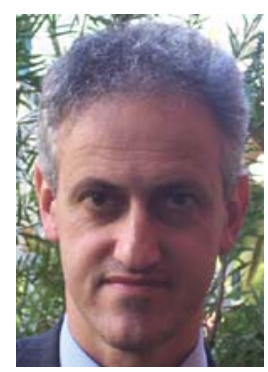

ITALIANO, GIUSEPPE

University of Rome Rome

Italy

\section{Stable Marriage Problems} Exact Algorithms

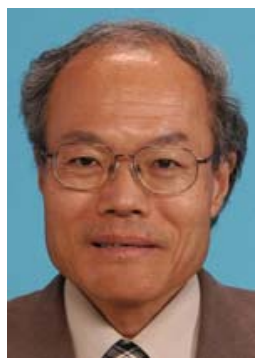

IWAMA, KAZUO

Kyoto University

Kyoto

Japan

\section{Approximation Algorithms}

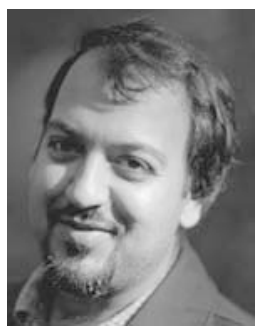

Khanna, Sanjeev

University of Pennsylvania

Philadelphia, PA

USA

\section{Graph Algorithms}

Combinatorial Optimization Approximation Algorithms

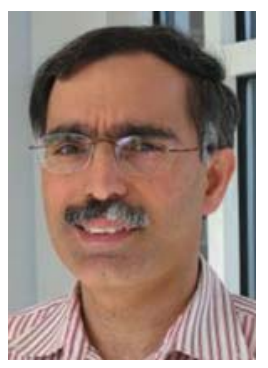

KhUller, SAmir

University of Maryland

College Park, MD

USA 
Compressed Text Indexing Computational Biology

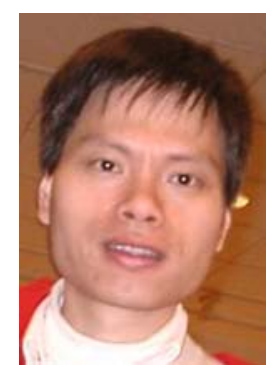

LAM, TAK-WAK

University of Hong Kong Hong Kong

China

\section{Mobile Computing}

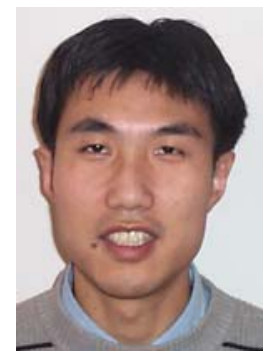

LI, XIANG-YANG

Illinois Institute of Technology

Chicago, IL

USA

\section{Geometric Networks}

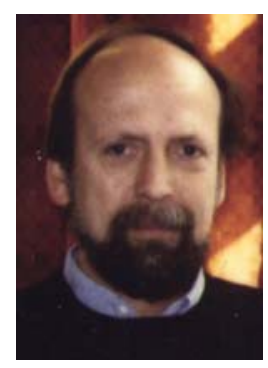

LINGAS, ANDRZEJ

Lund University

Lund

Sweden

\section{String Algorithms and Data Structures Compression of Text Data Structures}

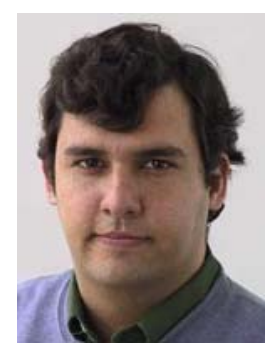

NAvarro, Gonzalo

University of Chile

Santiago

Chile

\section{Parameterized and Exact Algorithms}

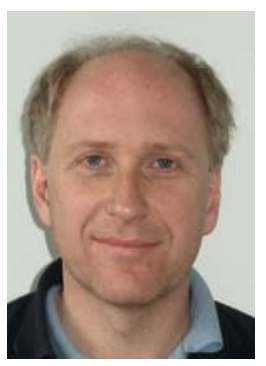

NeIdermeier, Rolf University of Jena Jena

Germany

\section{Probabilistic Algorithms} Average Case Analysis

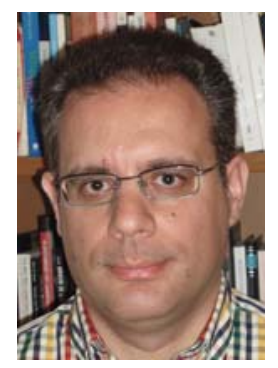

Nikoletseas, Sotiris

Patras University

Patras

Greece 


\section{Graph Algorithms}

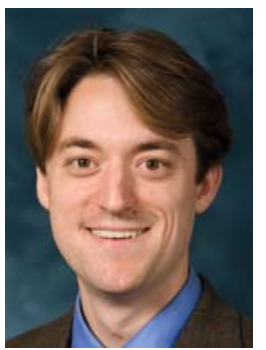

PetTie, Seth

University of Michigan

Ann Arbor, MI

USA

\section{Scheduling Algorithms}

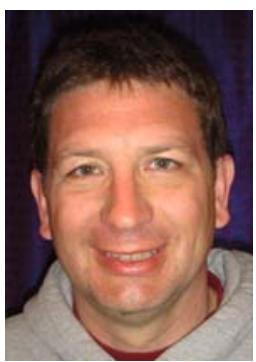

PRUHS, KIRK

University of Pittsburgh

Pittsburgh, PA

USA

\section{Distributed Algorithms}

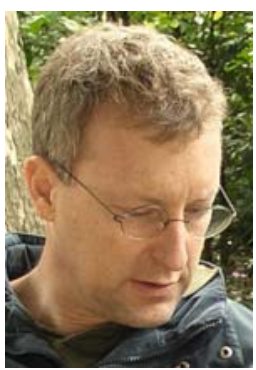

Rajsbaum, Sergio

National Autonomous University of Mexico Mexico City

Mexico

\section{Graph Algorithms}

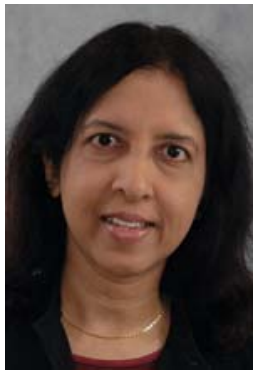

RAMACHANDRAN, VIJAYA University of Texas, Austin Austin, TX

USA

\section{Algorithm Engineering}

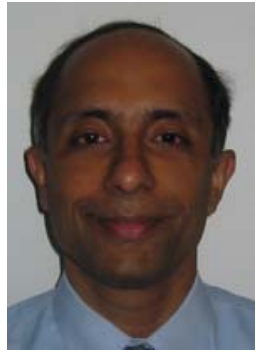

RAMAN, RAJEEV

University of Leicester

Leicester

UK

\section{Computational Learning Theory}

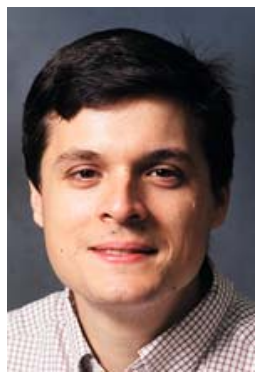

SERVEDIO, Rocco

Columbia University

New York, NY

USA 
Probabilistic Algorithms Average Case Analysis

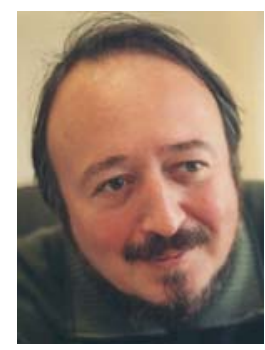

SPIRAKIS, PAVlOS (PAUL)

Patras University

Patras

Greece

\section{Scheduling Algorithms}

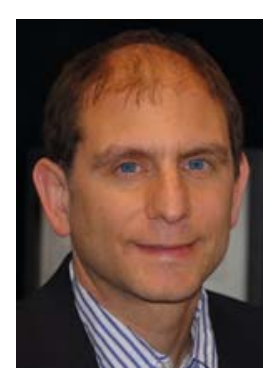

Stein, Clifford

Columbia University New York, NY

USA

\section{VLSI CAD Algorithms}

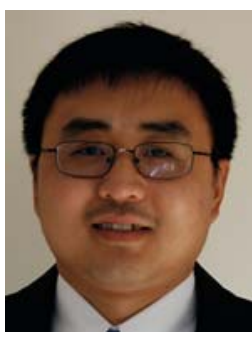

Zhou, Hai

Northwestern University

Evanston, IL

USA 


\section{List of Contributors}

\section{Aardal, Karen}

CWI

Amsterdam

The Netherlands

Eindhoven University of Technology

Eindhoven

The Netherlands

AKAVIA, ADI

MIT

Cambridge, MA

USA

Albers, Susanne

University of Freiburg

Freiburg

Germany

Alicherry, Mansoor

Bell Labs

Murray Hill, NJ

USA

Alon, NogA

Tel-Aviv University

Tel-Aviv

Israel

Altschul, Stephen F.

The Rockefeller University

New York, NY

USA

MIT

Cambridge, MA

USA

Aluru, SRINivas

Iowa State University

Ames, IA

USA

AMBAINIS, ANDRIS

University of Latvia

Riga

Latvia
AMBÜHL, CHRISTOPH

University of Liverpool

Liverpool

UK

AMIR, AMIHOOD

Bar-Ilan University

Ramat-Gan

Israel

Asodi, Vera

California Institute of Technology

Pasadena, CA

USA

Auer, Peter

University of Leoben

Leoben

Austria

AZIZ, ADNAN

University of Texas

Austin, TX

USA

Babaioff, Moshe

Microsoft Research, Silicon Valley

Mountain View, CA

USA

BADER, DAVID A.

Georgia Institute of Technology

Atlanta, GA

USA

BAEZA-YATES, RicARDO

University of Chile

Santiago

Chile

BANSAL, NIKHIL

IBM

Yorktown Heights, NY

USA 


\author{
BARBAY, JÉRÉMY \\ University of Chile \\ Santiago \\ Chile
}

BARUAH, SANJOY

University of North Carolina

Chapel Hill, NC

USA

BASWANA, SURENDER

IIT Kanpur

Kanpur

India

BECCHETTI, LUCA

University of Rome

Rome

Italy

Beimel, Amos

Ben-Gurion University

Beer Sheva

Israel

BÉKÉSI, JÓZSEF

Juhász Gyula Teachers Training College

Szeged

Hungary

BERgadano, Francesco

University of Torino

Torino

Italy

BERRY, VINCENT

LIRMM, University of Montpellier

Montpellier

France

BHATIA, RANDEEP

Bell Labs

Murray Hill, NJ

USA

BJÖRKLUND, ANDREAS

Lund University

Lund

Sweden

Blanchette, Mathieu

McGill University

Montreal, QC

Canada
BLÄSER, MARKUS

Saarland University

Saarbrücken

Germany

BodlaEnder, Hans L.

University of Utrecht

Utrecht

The Netherlands

BorRadAile, GLENCORA

Brown University

Providence, RI

USA

BShOUty, NADER H.

Technion

Haifa

Israel

Buchsbaum, ADAm L.

AT\&T Labs, Inc.

Florham Park, NJ

USA

Busch, Costas

Lousiana State University

Baton Rouge, LA

USA

Bu, Tian-Ming

Fudan University

Shanghai

China

BYRKA, JAROSLAW

CWI

Amsterdam

The Netherlands

Eindhoven University of Technology

Eindhoven

The Netherlands

CAI, MAO-CHENG

Chinese Academy of Sciences

Beijing

China

Calinescu, Gruia

Illinois Institute of Technology

Chicago, IL

USA

Cechlárová, Katarína

P.J. Šafárik University

Košice

Slovakia 
Chan, Chee-Yong

National University of Singapore

Singapore

Singapore

Chandra, Tushar DeEpaK

IBM Watson Research Center

Yorktown Heights, NY

USA

CHAO, KUN-MAO

National Taiwan University

Taipei

Taiwan

Charron-Bost, Bernadette

The Polytechnic School

Palaiseau

France

CHATZIGIANNAKIS, IOANNIS

University of Patras and Computer Technology Institute

Patras

Greece

CHAWLA, SHUCHI

University of Wisconsin-Madison

Madison, WI

USA

CHEKURI, CHANDRA

University of Illinois, Urbana-Champaign

Urbana, IL

USA

Chen, DAnny Z.

University of Notre Dame

Notre Dame, IN

USA

Cheng, XiUZhen

The George Washington University

Washington, D.C.

USA

CHEN, JIANER

Texas A\&M University

College Station, TX

USA

Chen, XI

Tsinghua University

Beijing, Beijing

China
Chin, Francis

University of Hong Kong

Hong Kong

China

Chowdhury, Rezaul A.

University of Texas at Austin

Austin, TX

USA

Christodoulou, George

Max-Planck-Institute for Computer Science

Saarbruecken

Germany

Chrobak, Marek

University of California at Riverside

Riverside, CA

USA

CHU, CHris

Iowa State University

Ames, IA

USA

Chu, XiaOWen

Hong Kong Baptist University

Hong Kong

China

Chuzhoy, Julia

Toyota Technological Institute

Chicago, IL

USA

CONG, JASON

UCLA

Los Angeles, CA

USA

COWEN, LENORE J.

Tufts University

Medford, MA

USA

CRISTIANINI, NELlO

University of Bristol

Bristol

UK

Crochemore, MaXime

King's College London

London

UK

University of Paris-East

Paris

France 
CSÜRÖS, MIKLÓS

University of Montreal

Montreal, QC

Canada

Czumaj, ARTur

University of Warwick

Coventry

UK

DASGUPTA, BHASKAR

University of Illinois at Chicago

Chicago, IL

USA

Défago, Xavier

Japan Advanced Institute of Science and Technology (JAIST)

Ishikawa

Japan

DEMAINE, ERIK D.

MIT

Cambridge, MA

USA

Demetrescu, Camil

University of Rome

Rome

Italy

Deng, Ping

University of Texas at Dallas

Richardson, TX

USA

Deng, Xiaotie

City University of Hong Kong

Hong Kong

China

DESPER, RICHARD

University College London

London

UK

DICK, ROBERT

Northwestern University

Evanston, IL

USA

DiNG, YUZHENG

Synopsys Inc.

Mountain View, CA

USA
DOM, MiCHAEL

University of Jena

Jena

Germany

Dubhashi, Devdatt

Chalmers University of Technology and Gothenburg

University

Gothenburg

Sweden

DU, DING-ZHU

University of Dallas at Texas

Richardson, TX

USA

EDMONDS, JEFF

York University

Toronto, ON

Canada

EFraimidis, PAVlos

Democritus University of Thrace

Xanthi

Greece

EFThymiou, Charilaos

University of Patras

Patras

Greece

Elkin, Michael

Ben-Gurion University

Beer-Sheva

Israel

EPSTEIN, LEAH

University of Haifa

Haifa

Israel

ERICKSON, BRUCE W.

The Rockefeller University

New York, NY

USA

EVEN-DAR, EyaL

University of Pennsylvania

Philadelphia, PA

USA

FAGERBERG, RolF

University of Southern Denmark

Odense

Denmark 
FAKCHAROENPHOL, JiTTAT

Kasetsart University

Bangkok

Thailand

FANG, QIZHI

Ocean University of China

Qingdao

China

Fatourou, Panagiota

University of Ioannina

Ioannina

Greece

Feldman, Jonathan

Google, Inc.

New York, NY

USA

Feldman, Vitaly

Harvard University

Cambridge, MA

USA

Fernau, Henning

University of Trier

Trier

Germany

Ferragina, PaOlo

University of Pisa

Pisa

Italy

FeUerstein, Esteban

University of Buenos Aires

Buenos Aires

Argentina

FISHER, NATHAN

University of North Carolina

Chapel Hill, NC

USA

FLAXMAN, ABRAHAM

Microsoft Research

Redmond, WA

USA

FLEISCHER, RUdOLF

Fudan University

Shanghai

China
FOMIN, FEDOR

University of Bergen

Bergen

Norway

FOTAKIS, DIMITRIS

University of the Aegean

Samos

Greece

FrIEDER, OPHIR

Illinois Institute of Technology

Chicago, IL

USA

FÜRER, MARTIN

The Pennsylvania State University

University Park, PA

USA

Gagie, Travis

University of Eastern Piedmont

Alessandria

Italy

GALAMBOS, GÁBOR

Juhász Gyula Teachers Training College

Szeged

Hungary

GAO, JIE

Stony Brook University

Stony Brook, NY

USA

Garay, JUAN

Bell Labs

Murray Hill, NJ

USA

Garofalakis, Minos

University of California - Berkeley

Berkeley, CA

USA

Gascuel, Olivier

National Scientific Research Center

Montpellier

France

GĄSIENIEC, LESZEK

University of Liverpool

Liverpool

UK 
GIANCARLO, RAFFAELE

University of Palermo

Palermo

Italy

Goldberg, ANDREW V.

Microsoft Research - Silicon Valley

Mountain View, CA

USA

GRAMM, JENS

Tübingen University

Tübingen

Germany

Grover, LOV K.

Bell Labs

Murray Hill, NJ

USA

Gudmundsson, JOACHIM

National ICT Australia Ltd

Alexandria

Australia

Guerraoui, RACHID

EPFL

Lausanne

Switzerland

GUO, JIONG

University of Jena

Jena

Germany

GURUSWAMI, VENKATESAN

University of Washington

Seattle, WA

USA

HAJIAGHAYI, MOHAMMADTAGHI

University of Pittsburgh

Pittsburgh, PA

USA

HALLGREN, SEAN

The Pennsylvania State University

University Park, PA

USA

HALPERIN, DAN

Tel-Aviv University

Tel Aviv

Israel
HARIHARAN, RAMESH

Strand Life Sciences

Bangalore

India

Hellerstein, Lisa

Polytechnic University

Brooklyn, NY

USA

HE, MENG

University of Waterloo

Waterloo, ON

Canada

HenZINGER, MONIKA

Google Switzerland \& Ecole Polytechnique Federale de Lausanne (EPFL)

Lausanne

Switzerland

HERLIHY, MAURICE

Brown University

Providence, RI

USA

Herman, Ted

University of Iowa

Iowa City, IA

USA

He, XIN

University at Buffalo The State University of New York Buffalo, NY

USA

HiRsCH, EDWARD A.

Steklov Institute of Mathematics at St. Petersburg

St. Petersburg

Russia

HON, WING-KAI

National Tsing Hua University

Hsin Chu

Taiwan

Howard, Paul G.

Microway, Inc.

Plymouth, MA

USA

HUANG, LI-SHA

Tsinghua University

Beijing, Beijing

China 
HUANG, YAOCUN

University of Texas at Dallas

Richardson, TX

USA

HÜFFNER, FALK

University of Jena

Jena

Germany

HusfeldT, THORE

Lund University

Lund

Sweden

ILIE, LUCIAN

University of Western Ontario

London, $\mathrm{ON}$

Canada

IRVING, ROBERT W.

University of Glasgow

Glasgow

UK

ITAI, ALON

Technion

Haifa

Israel

ITALIANO, GIUSEPPE F.

University of Rome

Rome

Italy

IWAMA, KAZUO

Kyoto University

Kyoto

Japan

JACKSON, JEFFREY C.

Duquesne University

Pittsburgh, PA

USA

JACOB, RIKO

Technical University of Munich

Munich

Germany

JAIN, RAHUL

University of Waterloo

Waterloo, ON

Canada
JANSSON, JESPER

Ochanomizu University

Tokyo

Japan

JIANG, TAO

University of California at Riverside

Riverside, CA

USA

JOHNSON, DAVID S.

AT\&T Labs

Florham Park, NJ

USA

KAJITANi, YoJI

The University of Kitakyushu

Kitakyushu

Japan

KAPORIS, ALEXIS

University of Patras

Patras

Greece

Karakostas, GeOrGe

McMaster University

Hamilton, ON

Canada

KÄRKKÄINEN, JUHA

University of Helsinki

Helsinki

Finland

KeLleRER, HANS

University of Graz

Graz

Austria

Kennings, ANDrew A.

University of Waterloo

Waterloo, ON

Canada

KeUtZER, KURT

University of California at Berkeley

Berkeley, CA

USA

KHULLER, SAMIR

University of Maryland

College Park, MD

USA 
KIM, JIN WOOK

HM Research

Seoul

Korea

KIM, YoO-AH

University of Connecticut

Storrs, CT

USA

KING, VALERIE

University of Victoria

Victoria, BC

Canada

KIROUSIS, LEFTERIS

University of Patras

Patras

Greece

KIVINEN, JYRKI

University of Helsinki

Helsinki

Finland

KLEIN, RolF

University of Bonn

Bonn

Germany

KLIVANS, ADAM

University of Texas at Austin

Austin, TX

USA

KonJeVod, Goran

Arizona State University

Tempe, AZ

USA

KONTOGIANNIS, SPYROS

University of Ioannina

Ioannina

Greece

KRANAKIS, EVANGELOS

Carleton

Ottawa, ON

Canada

KRATSCh, DieTer

Paul Verlaine University

Metz

France
Krauthgamer, Robert

Weizmann Institute of Science

Rehovot

Israel

IBM Almaden Research Center

San Jose, CA

USA

KRIZANC, DANNY

Wesleyan University

Middletown, CT

USA

KRYSTA, PIOTR

University of Liverpool

Liverpool

UK

Kucherov, Gregory

LIFL and INRIA

Villeneuve d'Ascq

France

KUHN, FABIAN

ETH Zurich

Zurich

Switzerland

KUMAR, V.S. ANIL

Virginia Tech

Blacksburg, VA

USA

Kushilevitz, Eyal

Technion

Haifa

Israel

LAM, TAK-WAH

University of Hong Kong

Hong Kong

China

LANCIA, GIUSEPPE

University of Udine

Udine

Italy

LANDAU, GAD M.

University of Haifa

Haifa

Israel

LANDAU, ZEPH

City College of CUNY

New York, NY

USA 
LANGBERG, MiCHAEL

The Open University of Israel Raanana

Israel

LAVI, RoN

Technion

Haifa

Israel

LECROQ, THIERRY

University of Rouen

Rouen

France

LEE, JAMES R.

University of Washington

Seattle, WA

USA

LEONARDI, STEFANO

University of Rome

Rome

Italy

LEONe, Pierre

University of Geneva

Geneva

Switzerland

LEUNG, HENRY

MIT

Cambridge, MA

USA

LEVCOPOUlos, CHRISTOS

Lund University

Lund

Sweden

Lewenstein, Moshe

Bar-Ilan University

Ramat-Gan

Israel

LI, LI (ERRAN)

Bell Labs

Murray Hill, NJ

USA

LI, MING

University of Waterloo

Waterloo, ON

Canada
LI, MinMing

City University of Hong Kong

Hong Kong

China

LINGAS, ANDRZEJ

Lund University

Lund

Sweden

LI, XIANG-YANG

Illinois Institue of Technology

Chicago, IL

USA

LU, CHIN LUNG

National Chiao Tung University

Hsinchu

Taiwan

LYNGS $\varnothing$, RUNE B.

Oxford University

Oxford

UK

MA, BIN

University of Western Ontario

London, $\mathrm{ON}$

Canada

MAHDIAN, MOHAMMAD

Yahoo! Research

Santa Clara, CA

USA

MÄKINEN, VELI

University of Helsinki

Helsinki

Finland

MALKhI, DAHLia

Microsoft, Silicon Valley Campus

Mountain View, CA

USA

MANASSE, MARK S.

Microsoft Research

Mountain View, CA

USA

Manlove, David F.

University of Glasgow

Glasgow

UK 
MANZINI, GIOVANNI

University of Eastern Piedmont

Alessandria

Italy

Marathe, Madhav V.

Virginia Tech

Blacksburg, VA

USA

Marchetti-Spaccamela, Alberto

University of Rome

Rome

Italy

MARKOV, IGOR L.

University of Michigan

Ann Arbor, MI

USA

MCGeoch, CATHerine C.

Amherst College

Amherst, MA

USA

MCGeOCH, Lyle A.

Amherst College

Amherst, MA

USA

MCKAY, BRENDAN D.

Australian National University

Canberra, ACT

Australia

Mendel, MANOR

The Open University of Israel

Raanana

Israel

MESTRE, JULiÁN

University of Maryland

College Park, MD

USA

Micciancio, DANiEle

University of California, San Diego

La Jolla, CA

USA

MikLÓS, IsTVÁN

Eötvös Lóránd University

Budapest

Hungary
MirRoKNI, VAHAB S.

Microsoft Research

Redmond, WA

USA

MiYAZAKI, SHUICHI

Kyoto University

Kyoto

Japan

Moffat, Alistair

University of Melbourne

Melbourne, VIC

Australia

MOIR, MARK

Sun Microsystems Laboratories

Burlington, MA

USA

MOR, TAL

Technion

Haifa

Israel

Mosca, Michele

University of Waterloo

Waterloo, ON

Canada

St. Jerome's University

Waterloo, ON

Canada

Moscibroda, Thomas

Microsoft Research

Redmond, WA

USA

MUCHA, MARCIN

Institute of Informatics

Warsaw

Poland

Munagala, Kamesh

Duke University

Durham, NC

USA

MUNRO, J. IAN

University of Waterloo

Waterloo, ON

Canada 
NA, JOONG CHAE

Sejong University

Seoul

Korea

NARASIMHAN, GIRI

Florida International University

Miami, FL

USA

NAVARro, GonZalo

University of Chile

Santiago

Chile

NAYAK, ASHWIN

University of Waterloo and Perimeter Institute for Theoretical Physics

Waterloo, ON

Canada

Newman, Alantha

Max-Planck Institute for Computer Science Saarbrücken

Germany

Niedermeier, RolF

University of Jena

Jena

Germany

NikOletseas, SOTIRIS

University of Patras

Patras

Greece

Окамото, Yoshio

Toyohashi University of Technology

Toyohashi

Japan

OKUn, MichaEL

Weizmann Institute of Science

Rehovot

Israel

Pagh, Rasmus

IT University of Copenhagen

Copenhagen

Denmark

Panagoroulou, Panagiota

Research Academic Computer Technology Institute

Patras

Greece
PANIGRAHI, DEBMALYA

MIT

Cambridge, MA

USA

Pan, Peichen

Magma Design Automation, Inc.

Los Angeles, CA

USA

PAPADOPOULOU, Vicky

University of Cyprus

Nicosia

Cyprus

PARK, KUnSOO

Seoul National University

Seoul

Korea

PARTHASARATHY, SRINIVASAN

IBM T.J. Watson Research Center

Hawthorne, NY

USA

PĂtraşCu, Mihai

MIT

Cambridge, MA

USA

PATT-SHAMIR, BOAZ

Tel-Aviv University

Tel-Aviv

Israel

PATURI, RAMAMOHAN

University of California at San Diego

San Diego, CA

USA

PELC, ANDRZeJ

University of Québec-Ottawa

Gatineau, QC

Canada

Pettie, Seth

University of Michigan

Ann Arbor, MI

USA

POWELL, OLIVIER

University of Geneva

Geneva

Switzerland 
PRAKASH, AMIT

Microsoft, MSN

Redmond, WA

USA

PRUHS, KIRK

University of Pittsburgh

Pittsburgh, PA

USA

PRZYTYCKA, TERESA M.

$\mathrm{NIH}$

Bethesda, MD

USA

PudLÁK, PAVEL

Academy of Science of the Czech Republic

Prague

Czech Republic

RAGHAVACHARI, BALAJI

University of Texas at Dallas

Richardson, TX

USA

RAHMAN, NAILA

University of Leicester

Leicester

UK

RAJARAMAN, RAJMOHAN

Northeastern University

Boston, MA

USA

RAJSBAum, SERGIO

National Autonomous University of Mexico

Mexico City

Mexico

RAMACHANDRAN, VIJAYA

University of Texas at Austin

Austin, TX

USA

RAMAN, RAJEEV

University of Leicester

Leicester

UK

RAMOS, EDGAR

National University of Colombia

Medellín

Colombia
RAO, SATISH

University of California at Berkeley

Berkeley, CA

USA

RAO, S. SRINIVASA

IT University of Copenhagen

Copenhagen

Denmark

RAPTOPOUlOS, CHRISTOFOROS

University of Patras

Patras

Greece

RASTOGI, RAJEEV

Lucent Technologies

Murray Hill, NJ

USA

RATSABY, JOEL

Ariel University Center of Samaria

Ariel

Israel

RAVINDRAN, KAUSHIK

University of California at Berkeley

Berkeley, CA

USA

RAYNAL, MiCHEL

University of Rennes 1

Rennes

France

REICHARDT, BEN W.

California Institute of Technology

Pasadena, CA

USA

RENNER, RENATO

Institute for Theoretical Physics

Zurich

Switzerland

RICCI, ELISA

University of Perugia

Perugia

Italy

Richter, Peter

Rutgers, The State University of New Jersey

Piscataway, NJ

USA 
ROLIM, JOSÉ

University of Geneva

Geneva

Switzerland

ROSAMOND, FRANCES

University of Newcastle

Callaghan, NSW

Australia

RÖTTELER, MARTIN

NEC Laboratories America

Princeton, NJ

USA

RUBINFELD, RONITT

MIT

Cambridge, MA

USA

RUDRA, ATRI

University at Buffalo, State University of New York Buffalo, NY

USA

RUPPERT, ERIC

York University

Toronto, ON

Canada

RYTTER, WOJCIECH

Warsaw University

Warsaw

Poland

SAHINALP, S. CENK

Simon Fraser University

Burnaby, BC

USA

SAKS, MiCHAEL

Rutgers, State University of New Jersey

Piscataway, NJ

USA

SCHÄFER, GUIDO

Technical University of Berlin

Berlin

Germany

SCHIPER, ANDRÉ

EPFL

Lausanne

Switzerland
SCHMIDT, MARKUS

University of Freiburg

Freiburg

Germany

SCHultes, Dominik

University of Karlsruhe

Karlsruhe

Germany

SEN, PRANAB

Tata Institute of Fundamental Research

Mumbai

India

SEN, SANDEEP

IIT Delhi

New Delhi

India

SERNA, MARIA

Technical University of Catalonia

Barcelona

Spain

SERVEDIO, Rocco

Columbia University

New York, NY

USA

Sethuraman, Jay

Columbia University

New York, NY

USA

SHALEV-SHWARTZ, SHAI

Toyota Technological Institute

Chicago, IL

USA

SHARMA, VIKRAM

New York University

New York, NY

USA

SHI, YAOYUN

University of Michigan

Ann Arbor, MI

USA

Shragowitz, Eugene

University of Minnesota

Minneapolis, $\mathrm{MN}$

USA 
Sitters, René A.

Eindhoven University of Technology

Eindhoven

The Netherlands

SMID, MiChIEL

Carleton University

Ottawa, ON

Canada

SoKol, DiNA

Brooklyn College of CUNY

Brooklyn, NY

USA

SONG, WEN-ZHAN

Washington State University

Vancouver, WA

USA

SPeckmann, Bettina

Technical University of Eindhoven

Eindhoven

The Netherlands

SPIRAKIS, PAUL

Patras University

Patras

Greece

SRINIVASAN, ARAVIND

University of Maryland

College Park, MD

USA

SRINIVASAn, Venkatesh

University of Victoria

Victoria, BC

Canada

Stee, Rob VAN

University of Karlsruhe

Karlsruhe

Germany

Stølting Brodal, Gerth

University of Aarhus

Århus

Denmark

Stoye, Jens

University of Bielefeld

Bielefeld

Germany
SU, CHANG

University of Liverpool

Liverpool

UK

SUN, ARIES WEI

City University of Hong Kong

Hong Kong

China

Sundararajan, ViJaY

Texas Instruments

Dallas, TX

USA

SUNG, WING-KIN

National University of Singapore

Singapore

Singapore

SVIRIDENKO, MAXIM

IBM

Yorktown Heights, NY

USA

SZEgEDY, MARIO

Rutgers, The State University of New Jersey

Piscataway, NJ

USA

SZEIDER, STEFAN

Durham University

Durham

UK

TAKAOKA, TADAO

University of Canterbury

Christchurch

New Zealand

TAKeda, Masayuki

Kyushu University

Fukuoka

Japan

Talwar, KUnaL

Microsoft Research, Silicon Valley Campus Mountain View, CA

USA

TAMON, Christino

Clarkson University

Potsdam, NY

USA 
TAMURA, AKIHISA

Keio University

Yokohama

Japan

TANNIER, ERIC

University of Lyon

Lyon

France

TAPP, ALAIN

University of Montréal

Montreal, QC

Canada

TATE, STEPHEN R.

University of North Carolina at Greensboro

Greensboro, NC

USA

TAUbenfeld, Gadi

Interdiciplinary Center Herzlia

Herzliya

Israel

Telikepalli, Kavitha

Indian Institute of Science

Bangalore

India

Terhal, BARbara M.

IBM Research

Yorktown Heights, NY

USA

Thilikos, Dimitrios

National and Kapodistrian University of Athens

Athens

Greece

TREvisan, LUCA

University of California at Berkeley

Berkeley, CA

USA

Tromp, JoHN

CWI

Amsterdam

Netherlands

UKKONEN, ESKO

University of Helsinki

Helsinki

Finland
VAHRENHOLD, JAN

Dortmund University of Technology

Dortmund

Germany

VARRICCHIO, STEFANO

University of Roma

Rome

Italy

Vialette, STÉPhane

University of Paris-East

Descartes

France

VilLANGER, YNGVE

University of Bergen

Bergen

Norway

VITÁNYI, PAUL

CWI

Amsterdam

Netherlands

VitTER, JefFrey SCOTT

Purdue University

West Lafayette, IN

USA

VÖCKING, BERTHOLD

RWTH Aachen University

Aachen

Germany

WANG, CHENGWEN CHRIS

Carnegie Mellon University

Pittsburgh, PA

USA

WANG, FENG

Arizona State University

Phoenix, AZ

USA

WANG, LUSHENG

City University of Hong Kong

Hong Kong

China

WANG, WEIZHAO

Google Inc.

Irvine, CA

USA 
WANG, YU

University of North Carolina at Charlotte

Charlotte, NC

USA

WAN, PEng-Jun

Illinois Institute of Technology

Chicago, IL

USA

WERNECK, RENATO F.

Microsoft Research Silicon Valley

La Avenida, CA

USA

WILLIAMS, RYAN

Carnegie Mellon University

Pittsburgh, PA

USA

WONG, MARTIN D. F.

University of Illinois at Urbana-Champaign

Urbana, IL

USA

Wong, Prudence

University of Liverpool

Liverpool

UK

Wu, WEILI

University of Texas at Dallas

Richardson, TX

USA

YANG, HoNGHUA HANNAH

Intel Corporation

Hillsboro

USA

YAP, CHEE K.

New York University

New York, NY

USA

YE, YIN-YU

Stanford University

Stanford, CA

USA

Yi, CHIH-WEI

National Chiao Tung University

Hsinchu City

Taiwan
YI, KE

Hong Kong University of Science and Technology

Hong Kong

China

YIU, S. M.

The University of Hong Kong

Hong Kong

China

YOKOO, MAKOTO

Kyushu University

Nishi-ku

Japan

Young, Evangeline F. Y.

The Chinese University of Hong Kong

Hong Kong

China

Young, NeAl E.

University of California at Riverside

Riverside, CA

USA

YUSTER, RAPHAEL

University of Haifa

Haifa

Israel

ZANE, FRANCIS

Lucent Technologies

Murray Hill, NJ

USA

ZAROLIAGIS, CHRISTOS

University of Patras

Patras

Greece

ZEH, NORBERT

Dalhousie University

Halifax, NS

Canada

ZHANG, LI

HP Labs

Palo Alto, CA

USA

ZHANG, LOUXIN

National University of Singapore

Singapore

Singapore 
ZHOU, HAI

Northwestern University

Evanston, IL

USA

ZiLles, SANDRA

University of Alberta

Edmonton, $\mathrm{AB}$

Canada
ZOLLINGER, AARON

University of California at Berkeley

Berkeley, CA

USA

ZWICK, URI

Tel-Aviv University

Tel-Aviv

Israel 